\title{
REMARKS ON THE ASYMPTOTIC PROPERTIES OF SOLUTIONS OF FREDHOLM INTEGRAL EQUATIONS*
}

\author{
By GEORGE R. BART (Mayfair College, Chicago)
}

A theorem on the large-argument asymptotic behavior of the solution of a translationtype of linear integral equation was recently obtained by Muki and Sternberg [1]. Presented herein is a lemma whose domain of application overlaps, in part, that of their theorem. When our lemma applies one is able to prove the existence of a solution of the type that their theorem presupposes. Our lemma provides a useful asymptotic bound for the solution of any regular Fredholm equation whenever, in the context of conventional Fredholm theory, the resolvent exists. The approach presented here was previously used to determine the asymptotic behavior of the solution of a Fredholm integral equation which arises in the theory of scattering of elementary particles [2].

Let us consider the Fredholm integral equation

$$
\varphi(x)=f(x)+\int_{0}^{\infty} K(x, s) \varphi(x) d s, \quad 0 \leq x<\infty .
$$

We presume that Fredholm theory applies and that 1 is not an eigenvalue of $K$. Then Eq. (1) has a solution which is unique. As a step towards obtaining the large-argument asymptotic behavior of this solution we present the following lemma.

LEMma. If in the regular Fredholm equation (1), 1 is not an eigenvalue of $K$, and if, as $x \rightarrow \infty$,

$$
\begin{aligned}
f(x) & =O\left[f_{A}(x)\right], \\
k(x) & =\left\{\int_{0}^{\infty}|K(x, s)|^{2} d s\right\}^{1 / 2}=O\left[k_{A}(x)\right], \\
K f(x) & =\int_{0}^{\infty} K(x, s) f(s) d s=O\left[K_{A} f(x)\right],
\end{aligned}
$$

then the solution $\varphi$ of (1) is bounded by

$$
\varphi(x)=O\left[f_{A}(x)\right]+O\left[K_{A}(x)\right]+O\left[k_{A}(x)\right] .
$$

The proof by means of the Schwarz inequality is almost trivial. Let $H$ be the resolvent, $1+H=(1-K)^{-1}$. Then

$$
\begin{aligned}
\varphi & =f+H f \\
& =f+K f+K H f
\end{aligned}
$$

and

$$
|\varphi| \leq|f|+|K f|+k \| H f||
$$

\footnotetext{
* Received February 5, 1972.
} 
where the double bars denote the norm of $H f$. Eq. (5) follows immediately from (2)-(4) and (7).

To apply the lemma, ordinarily one can determine $f_{A}, k_{A}$, and $K_{A} f$ from a knowledge of the asymptotic behavior of the given functions $f$ and $K$. The usefulness of the lemma comes from the fact that frequently one can use the bound (5) to extract from the right-hand side of (1) a specific form for the asymptotic behavior of $\varphi$. For example, let $K$ be the real-valued translation kernel of Muki and Sternberg,

$$
K(x, s)=\lambda_{1} G(x-s)+\lambda_{2} G(x+s),
$$

where $\lambda_{1}$ and $\lambda_{2}$ are real constants and $G(-x)=G(x)$. They hypothesize that $G$ and $f$ are continuous on $(0, \infty)$ and $[0, \infty)$, respectively. Also they suppose that $G$ is absolutely integrable on $[0, \infty)$ and that as $x \rightarrow \infty$

$$
\begin{array}{lll}
G(x)=a x^{-m}+O\left(x^{-m-1}\right), & a \neq 0, & 1<m<\infty, \\
f(x)=b x^{-n}+o\left(x^{-n}\right), & b \neq 0, & 0<n<\infty .
\end{array}
$$

Their theorem states that if there is a solution $\varphi$ of (1) such that $\varphi$ is continuous on $[0, \infty)$ and as $x \rightarrow \infty$

$$
\varphi(x)=\alpha x^{-\mu}+o\left(x^{-\mu}\right), \quad \alpha \neq 0, \quad 0 \leq \mu<\infty .
$$

then (a) $m \leq n$ implies $\mu \geq m$ or (b) $m>n$ implies $\mu=n, \alpha=b / \beta$, provided

$$
\beta=1-2 \lambda_{1} \int_{0}^{\infty} G(s) d s \neq 0 .
$$

Let us show with the aid of our lemma that if in addition to the Muki-Sternberg assumptions we suppose that $\lambda_{1}=0, n>\frac{1}{2}$, and that 1 is not an eigenvalue of $K$, then a $\varphi$ with the properties they suppose, including property (11), does in fact exist. A natural way to investigate (1) with the kernel (8) is by using the Weiner-Hopf technique. For simplicity, however, we take $\lambda_{1}=0$ so that Fredholm theory applies also. It is easy to see that if $\varphi$ is continuous on $[0, \infty)$, then $K \varphi$ is also. In applying conventional Fredholm theory we look for the solution of (1) in the space $C^{2}$ of continuous functions which are square integrable on $[0, \infty)$. We take $n>\frac{1}{2}$ so that $f$ is in $C^{2}$. For $K$ to be a $C^{2}$ kernel it is required that $\lambda_{1}=0$. To see this, suppose $\lambda_{1} \neq 0$ and observe that

$$
k^{2}(x)=2 \lambda_{1}^{2} \int_{0}^{\infty} G^{2}(t) d t-\left(\lambda_{1}^{2}-\lambda_{2}^{2}\right) \int_{x}^{\infty} G^{2}(t) d t+2 \lambda_{1} \lambda_{2} \int_{x}^{\infty} G(t) G(t-2 x) d t .
$$

As $x \rightarrow \infty$ the second integral in (13) is $O\left(x^{-2 m+1}\right)$ because of (9). The last integral in (13) can be shown to be $O\left(x^{-m}\right)$. But the first term in (13) is a non-zero constant. This implies that the integral of $k^{2}$ over $[0, \infty)$ diverges. Hence $K$ is a $C^{2}$ kernel if, and only if, $\lambda_{1}=0$.

Suppose $\lambda_{1}=0$. One can verify with the aid of a lemma given by Muki and Sternberg that now

$$
\begin{aligned}
f_{A}(x) & =x^{-n}, \\
k_{A}(x) & =x^{-m+1 / 2}, \\
K_{A} f(x) & =x^{-\mu}, \quad \mu=\min (m, n),
\end{aligned}
$$


with $\frac{1}{2}<n<\infty$ and $1<m<\infty$. Hence by Fredholm theory and our lemma, the unique, continuous, square-integrable solution $\varphi$ of (1) has the asymptotic bound

$$
\varphi(x)=O\left(x^{-\mu}\right), \quad \mu=\min \left(n, m-\frac{1}{2}\right)>\frac{1}{2} .
$$

This bound and the continuity of $\varphi$ are sufficient to carry out the steps of the MukiSternberg lemma which imply that

$$
\begin{aligned}
\int_{0}^{\infty} \varphi(s) G(x+s) d s & =a c x^{-m}+o\left(x^{-m}\right), & & \text { if } \quad \mu \geq m, \\
& =o\left(x^{-\mu}\right), & & \text { if } \quad \mu<m,
\end{aligned}
$$

where $c=\int_{0}^{\infty} \varphi(s) d s$. Thus, since (17) implies that $\mu$ is always less than $m$, from the right-hand side of (1) one gets the same asymptotic form for $\varphi$ as in the Muki-Sternberg theorem with $\lambda_{1}=0$.

The preceding approach can be useful in cases involving more delicate bounds than are encountered here. See, for example, [2] where many of the integrals converge only by virtue of logarithmic factors.

In conclusion let me add that three recent Russian works on the asymptotic behavior of integral equations of the first kind are reviewed in Mathematical Reviews 42. Also I wish to thank R. Warnock for helpful discussions.

\section{REFERENCES}

[1] R. Muki and E. Sternberg, Note on an asymptotic property of solutions to a class of Fredholm integral equations, Quart. Appl. Math. 28, 277-281 (1970)

[2] G. R. Bart and R. L. Warnock, Asymptotics of partial waves in Regge theory and constraints on partialwave subtraction constants, Phys. Rev. D3, 1429-1438 (1971) 